(2) Open Access Full Text Article

ORIGINAL RESEARCH

\title{
Postpartum depression screening in the Neonatal Intensive Care Unit: program development, implementation, and lessons learned
}

This article was published in the following Dove Press journal:

Journal of Multidisciplinary Healthcare

18 February 2016

Number of times this article has been viewed

\author{
Amanda S Cherry' \\ Ryan T Blucker' \\ Timothy S Thornberry ${ }^{2}$ \\ Carla Hetherington ${ }^{3}$ \\ Mary Anne McCaffree ${ }^{3}$ \\ Stephen R Gillaspy' \\ 'Department of Pediatrics, Section of \\ General and Community Pediatrics, \\ University of Oklahoma Health \\ Sciences Center, Oklahoma City, OK, \\ 2Department of Psychology, Morehead \\ State University, Morehead, KY, \\ ${ }^{3}$ Department of Pediatrics, College \\ of Medicine, University of Oklahoma, \\ Oklahoma City, OK, USA
}

Correspondence: Amanda S Cherry Department of Pediatrics, Section of General and Community Pediatrics, University of Oklahoma Health Sciences Center, 1200 Children's Ave, Suite I2400, Oklahoma City, OK 73I04, USA

$\mathrm{Tel}+\mathrm{I} 40527 \mid 4407$

Fax + I 405 27I 8709

Email amanda-cherry@ouhsc.edu
Objective: The aims of this project were to describe the development of a postpartum depression screening program for mothers of infants in the Neonatal Intensive Care Unit and assess the implementation of the screening program.

Methods: Screening began at 14 days postpartum and was implemented as part of routine medical care. A nurse coordinator facilitated communication with mothers for increasing screen completion, review of critical self-harm items, and making mental health referrals. During the 18-month study period, 385 out of 793 eligible mothers completed the screen.

Results: Approximately $36 \%$ of mothers had a positive screen that resulted in a mental health referral and an additional $30 \%$ of mothers had screening results indicating significant symptoms. Conclusion: Several barriers were identified, leading to adjustments in the screening process, and ultimately recommendations for future screening programs and research. Development of a postpartum depression screening process in the Neonatal Intensive Care Unit involves support, training, implementation, and coordination from administrators, medical staff, new mothers, and mental health specialists. Several predictable challenges to program development require ongoing assessment and response to these challenges.

Relevance: This study highlights the expanding role of the psychologist and behavioral health providers in health care to intervene as early as possible in the life of a child and family with medical complications through multidisciplinary program development and implementation, as well as key considerations for institutions initiating such a program.

Keywords: NICU, parental stress, barriers, postpartum depression, universal screening, pre term infants

\section{Introduction}

Postpartum depression (PPD) occurs in $\sim 15.7 \%$ of new mothers in the general population, ${ }^{1}$ and up to $80 \%$ of new mothers experience symptoms of depression such as fatigue and mood swings., ${ }^{2,3}$ Although considered to be the most common complication of childbirth, ${ }^{4,5}$ PPD is underidentified, underdiagnosed, and undertreated., ${ }^{4,6}$ Compared with mothers of healthy term infants, the rate of PPD increases to $28 \%-70 \%$ for mothers of infants admitted to the Neonatal Intensive Care Unit (NICU) setting. ${ }^{4,7}$ More specifically, mothers of preterm infants are $40 \%$ more likely to develop PPD than the general population..$^{5}$ Furthermore, mothers of infants admitted to the NICU are more likely to experience risk factors associated with PPD including relationship difficulties, family stress, and financial stress. ${ }^{8}$

The stressors experienced by mothers of infants in the NICU vary from those of term infants and occur at higher rates. ${ }^{9,10}$ For example, parents of NICU infants 
often experience high stress, feelings of helplessness, and uncertainty about how to interact with their neonate during the NICU stay. ${ }^{11-14}$ Additional risk factors for PPD specific within the NICU population have been suggested to include perception of the infant's illness severity, poor coping skills, increased parenting stress, and length of stay in the NICU. ${ }^{15}$ More specifically, perceptions of the infant's severity of illness has been suggested to be the strongest factor related to parental stress scores. ${ }^{16}$ Additionally, maternal factors such as marital status, stress from experiencing altered parental roles, and infant factors such as prolonged ventilation have been associated with increased depression. ${ }^{15}$

It has also been suggested that the duration of PPD is associated with delays in treatment, which are complicated by difficulties in early identification, one of the most challenging aspects of PPD. ${ }^{17}$ For example, the identification of PPD is often delayed as a result of mothers' feelings of shame and guilt, accompanied by feelings of being unable to care for their child, leading to reluctance in expressing their need for assistance. ${ }^{6,18}$ Frequently, mothers try to hide what they are feeling due to stigma associated with mental illness following childbirth, ${ }^{17}$ and perceptions of being unable to meet expectations of carrying out maternal roles. ${ }^{18}$ Additionally, in the NICU setting in particular, early identification can be complicated by the focus of care being on the infant as well as providers lacking expertise in diagnosis and treatment of adults. The depressed parent is typically not under the direct care of the NICU provider. ${ }^{19}$ In addition to provider discomfort in treating an individual not under direct care, there is parental discomfort in seeking assistance from providers for which they are dependent upon for their infant's care. ${ }^{20} \mathrm{By}$ default, the focus of the care in the NICU is primarily on the infant, rather than the mother, which can also lend the mother to being less likely to seek the social and emotional support of NICU providers. Many pediatricians feel responsible for recognizing maternal depression, but lack confidence and knowledge of how to do so. ${ }^{19}$ Although there is some merit to the argument that maternal depression is outside the scope of practice in the NICU, it is an important factor to consider regarding the discharge environment of the neonate. ${ }^{7}$

Higher prevalence of PPD and risk for significant consequences to the mother and infant justifies a call for increased depression screening in the NICU. It has been recommended that screening of mothers with infants in the NICU should occur routinely. ${ }^{21}$ Additionally, universal screening for PPD has been argued as an important part of every family assessment, given the increased risks inherent in the NICU environment. ${ }^{17}$ Screening mothers for PPD prior to their infants' discharge from the NICU may also be useful in recognizing those at highest risk for developing chronic depression. ${ }^{22}$ Routine screening has been suggested to normalize the process, enhance awareness, and increase the health care providers' competency and comfort level. ${ }^{17}$ Neonatal care providers have optimal opportunity for developing relationships with mothers of infants in the NICU as well as identifying symptoms of PPD in order to provide appropriate referrals and support. ${ }^{17}$ Additionally, infants in the NICU will likely have a significant length of stay allowing for reasonable time and opportunities to reach mothers for universal screening. ${ }^{7}$

At this time, PPD screening in the NICU is not considered standard of care. ${ }^{7}$ However, screening has been advocated for by multiple sources. ${ }^{23}$ Recommendations have been made for providers to assess and monitor for PPD symptoms throughout the first year following birth. ${ }^{5}$ The American Academy of Pediatrics has recommended screening of mothers at 1-, 2-, and 4-month visits. ${ }^{4,24}$ Further, PPD screening mechanisms are recommended to be available in all facilities serving pregnant women, new mothers, and newborns by the Association of Women's Health, Obstetric, and Neonatal Nurses. ${ }^{4}$ The implementation of screeners in general as well as in the NICU has been consistently supported by proven interventions. ${ }^{10}$

Previous studies have argued that screening for PPD is feasible in an outpatient setting, as well as effective in improving identification and treatment. ${ }^{19,25-27}$ However, screening for PPD in primary care settings is below $50 \% .{ }^{19}$ Previous studies have identified three primary types of barriers when putting outpatient PPD screening into place including patient-centered, physician-centered, and systems barriers. ${ }^{19}$ Patient-centered variables consist of cost and lack of insurance coverage, time constraints, social stigma, ${ }^{28,29}$ treatment compliance, and lack of access to care. ${ }^{28}$ Physician-centered barriers consist of time constraints, insufficient knowledge, ${ }^{28,30}$ competing demands, ${ }^{19,28}$ and fear of liability. ${ }^{19}$ System-based barriers have been suggested to consist of infrequent follow-up, lack of opportunities to monitor, and lack of integration of primary care and mental health services. ${ }^{19,28}$

In addition to the barriers identified in screening in an outpatient setting, mothers of NICU infants may have additional challenges. For example, it is well established that mothers of NICU infants are less likely to seek health care services. $^{7}$ Further, waiting for screening to occur in the outpatient setting is missing key opportunities to reach mothers of NICU infants at early, treatable stages. It has been previously suggested that mothers of preterm infants 
are particularly vulnerable to experiencing depression in the immediate postpartum period. ${ }^{5}$ Despite solid rationale for universal screening for PPD in NICU mothers, little is presented in the literature about the feasibility and challenges of such endeavors.

Mounts ${ }^{7}$ identified barriers to universal screening for PPD specific to the NICU population, which were consistent in types of barriers identified previously for the outpatient setting. Similar to outpatient barriers, the barriers identified included patient centered, physician-centered/staff-centered, and system-based barriers; however, some barriers varied by what was included in each type to reflect specific challenges within the NICU setting. For example, patient-centered barriers consisted of the mother's inability to disclose her feelings and lack of knowledge or acceptance of myths about PPD, as well as social stigma, access to services, and cost. Physician-centered and staff-centered barriers were suggested to include lack of time, unfamiliarity of screening instruments, concern about liability, and lack of community resources available for referrals for additional services. System-based barriers were suggested to include the lack of available and accessible mental health resources. ${ }^{7}$

The objectives of this study were to describe the implementation of depression screening using the Postpartum Depression Screening Scale (PDSS) in a NICU and identify barriers encountered in the process. Ultimately, the aim was to share lessons learned with others who are considering such a screening process, in order to enhance future endeavors in universal screening for PPD in mothers in the NICU.

\section{Methods}

This study reflects an 18-month screening period. Participants were recruited from a NICU in the south central region of the USA that has $\sim 1,200$ admissions yearly, with an average length of stay of 3 weeks, and a mortality rate of $10 \%-15 \%$. Premature infants as well as infants born at or near term are admitted and between $30 \%$ and $40 \%$ of admissions are transfers from other facilities. The project was a clinical demonstration project, thus mothers were not prospectively consented, but received routine clinical care. However, approval was obtained from the University of Oklahoma Health Sciences Center Institutional Review Board to assess the effectiveness of the project. Throughout the project, eligible participants were identified by the project coordinator following their infants' admission to the NICU. Screening was planned to start at 14 days postpartum and implemented as part of routine care. Initially, a project coordinator attempted to meet with NICU mothers to discuss the purpose and nature of the screening; due to difficulty making contact with mothers, nursing staff were asked to incorporate PPD screening into routine screening procedures (ie, the phenylketonuria [PKU] newborn screen) to ensure administration; connecting the PDSS to the PKU screening process was also not very effective. Thus, the final screening process included the coordinator placing the PDSS and an informational screening brochure in the room, along with an envelope and instructions to turn in the completed PDSS.

The PDSS $^{31}$ is a 35 -item self-report Likert scale used to assess symptoms of PPD. The PDSS is one of the most widely used screeners of $\mathrm{PPD}^{10,31}$ and has established validity and good internal consistency, 0.83-0.94. ${ }^{31}$ The PDSS was chosen for this study over other commonly used PPD screening tools such as the Edinburgh Postnatal Depression Scale (EPDS), specifically due to items with a focus on motherhood. Limitations of the EPDS include lack of evaluation regarding the context of a woman's experience as a new mother, including loss of control, loneliness, feelings of being unreal, loss of self, and difficulties with concentration. ${ }^{32}$ With specific interests in risk factors such as feelings of altered parental roles, inclusion of items focused on motherhood were important considerations. Although the PDSS has not been normed in the NICU population, it has been used by previous studies to assess maternal depression for mothers of NICU infants. ${ }^{11} \mathrm{McCabe}$ et $\mathrm{al}^{33}$ found the psychometric properties of the PDSS scores to be well supported with excellent estimates of internal consistency of total PDSS scores and adequate-to-good for subscale scores, with a NICU population. Additionally, Blucker et a ${ }^{10}$ found preliminary validation of a 15 -item variation of the PDSS within the NICU setting. Total PDSS score was used in the analyses to assess maternal report of PPD symptoms.

The coordinator facilitated further communication with mothers for increasing screen completion, review of critical self-harm items, and making mental health referrals. Mothers with positive screens were referred to the psychology team for diagnostic interviewing. Screening feedback forms were then given to mothers. The screening feedback forms included screening results and information about PPD and referral resources.

Descriptive statistics were used to demonstrate the utility of the PDSS in the NICU.

\section{Results}

During the 18-month study period, a total of 1,362 mothers had infants who were admitted in the NICU; of these, 793 $(58.2 \%)$ were in the NICU for at least 14 days and were 
therefore eligible for this study. A total of 385 out of 793 (48.5\%) eligible mothers completed the PDSS screen.

Regarding demographic characteristics, the mean age of the sample was 25.77 years ( standard deviation $=5.95$ ). Mothers of infants in the NICU were primarily Caucasian $(63 \%)$, married $(41 \%)$, or single (38\%), and had either a high school education $(43 \%)$ or had attended at least some college (42\%). Additional demographic characteristics for race, marital status, and education are provided in Table 1.

In regard to mental health variables, $24 \%$ of NICU mothers had a self-reported history of depression prior to delivery and about $22 \%$ reported previous depression treatment. Based on responses to the Postpartum Screening Scale, many of the women (36\%) received a positive screening for major PPD that resulted in a mental health referral. An additional 30\% showed significant symptoms on the PDSS and were considered "at risk," indicating the possible need for a referral to a mental health professional. Frequencies of all mental health variables within this sample are provided in Table 2.

A number of barriers to completing universal screening for PPD within the NICU population have been identified in the literature. ${ }^{7}$ Consistent with previous literature, the current study encountered several barriers to implementing a universal screening program. The majority of the barriers identified were influenced by patient-centered, provider-centered, and system-centered barriers. The primary barriers identified were within establishing contact, administration of the measure, and access to referrals. It has been previously suggested that barriers can be discussed within patient-centered, provider-centered, and system-centered types; however, the current project found that factors from each of these types of barriers were coexisting in each area identified and difficult

Table I Participant characteristics

\begin{tabular}{ll}
\hline Variable & Sample (n=385) \\
\hline Age in years (mean, standard deviation) & $25.77(5.95)$ \\
Race/ethnicity (\%) & \\
Caucasian & 62.6 \\
Black/African American & 15.3 \\
Native American/Alaskan Native & 8.3 \\
Hispanic/Latino & 7.3 \\
Other/unknown & 6.5 \\
Marital status (\%) & \\
Married & 40.5 \\
Single & 38.2 \\
Partnered & 12.5 \\
Divorced/separated & 2.9 \\
Other/unknown & 6.0 \\
Education (\%) & \\
Some college or higher & 42.3 \\
High school graduate & 42.9 \\
Less than high school graduate & 13.8 \\
\hline
\end{tabular}

Table 2 Sample mental health variables

\begin{tabular}{lll}
\hline Variables & $\begin{array}{l}\text { Frequency } \\
(\mathbf{n = 3 8 5 )}\end{array}$ & Rates (\%) \\
\hline History of depression & 94 & 24.4 \\
History of depression treatment & 83 & 21.6 \\
$\begin{array}{l}\text { Postpartum depression } \\
\quad \text { Positive screen for PPD }\end{array}$ & 137 & \\
$\quad$ Significant symptoms of PPD & 117 & 35.6 \\
$\quad$ Normal adjustment & 131 & 30.4 \\
\hline
\end{tabular}

Abbreviation: PPD, postpartum depression.

to separate out as each impacted the others. Thus, the barriers identified within the screening process, administration concerns, and access to referrals will be discussed from a multifactor perspective. Development of a PPD screening process in the NICU involves support, training, implementation, and coordination from administrators, medical staff, new mothers, and mental health specialists. Several predictable challenges to program development require ongoing assessment and response to these challenges.

\section{Barriers in establishing contact}

It has been previously suggested that PPD screening should be a multidisciplinary endeavor and should consider a range of providers available to administer screening. ${ }^{7}$ The current study initially planned for the project coordinator to contact mothers and complete screening at or beyond 14 days postpartum. However, it was quickly realized that it was difficult for the project coordinator to find mothers to administer the screener consistently. Some compounding factors included the size of the NICU, mothers not being in their infants' rooms at times of attempted contact, and the coordinator held a half-time position. Thus, due to both patient-centered and providercentered barriers, opportunities for contact and completion of the screening instrument were limited.

As a result of these difficulties, collaboration with the medical center nursing administration took place to incorporate the administration of the PDSS screener into their routine nursing duties at 2-week PKU newborn screening. The PDSS was attached to the PKU screen and nurses would ask mothers to complete the screen when the nurse completed the PKU screen. If the mother was not present at the time of PKU screen, the nurse would pin the PDSS to the infant's information board and remind the mother to complete the screen while visiting her infant. Once completed, the mother would give the screen to the nurse who placed the screen in a designated place for the coordinator. The coordinator routinely (twice per week) checked the completed screens and reviewed the self-harm items 
$(7,14,21,28$, and 35$)$. In the case of any critical items being positively endorsed, the coordinator notified the appropriate personnel per existing policy. Additionally, the coordinator would follow-up with any mothers who were given a screen but had not completed the screen. If the mother was present, the coordinator would give the screen to the mother to complete. Once completed, the mother would give the screen to the nurse and the same procedure was followed. The coordinator was on the unit each week to collect completed screens, review critical items, score the screens, follow-up with mothers who had not completed screens, and facilitate further assessment by the psychologist. Also, the coordinator kept a database where she would track the mothers' screening status.

It was believed that making the screening process part of other routine screening practices would allow for feasibility for nurses to administer the PPD screening in addition to their other nursing duties. However, due to the size of the unit and number of support staff involved in routine screening, connecting these two processes was found to be difficult. Thus, a system-centered barrier was also encountered. Thus, the final screening process was a combination of the first two processes. At 14 days postpartum, the coordinator placed the PDSS and an informational screening brochure in the room, along with an envelope and instructions to turn in the completed PDSS to a designated place. The coordinator again routinely (twice per week) checked the completed screens and reviewed the self-harm items $(7,14,21,28$, and 35$)$. If any critical items were positively endorsed then the coordinator would notify appropriate personnel per policy. Additionally, as in the previous process, the coordinator would follow-up with any mothers who were given a screen but had not completed the screen. Further, the coordinator would provide the mothers with either the positive or negative screening feedback form. These forms allowed mothers more immediate feedback on their screening results. This third and final strategy lowered the burden of time required by the project coordinator as well as the nursing staff, which addressed the provider-centered barrier. This final strategy also allowed for a more widespread attempt to contact. However, this strategy limited the interaction and relationship factor which has been previously associated with mothers' willingness to open up and express their experience of symptoms. ${ }^{17}$ Before mothers are willing to open up and share their negative thoughts and feelings, a safe and trusting environment is necessary. ${ }^{17}$ Thus, the lower report of clinically significant PPD results in comparison to previous studies may be explained by the use of the final strategy.
The current study supports the tenets of previous studies in the inclusion of the availability of multiple providers or disciplines, as well as, the importance of a relationship with the mother and a supportive NICU environment. As suggested by previous studies, the current study supports the importance of considering the mother's perception of her level and provider of support when deciding who to administer the screening. ${ }^{7}$ This process may lead to openness and willingness to engage in the screening process. Thus, considerations regarding availability of multiple providers/disciplines in the acts of making contact and establishing supportive relationships with mothers are key to future endeavors in universal screening for this population.

\section{Administrative barriers}

In addition to barriers in making contact and establishing a supportive relationship with mothers, there were some barriers identified in this study that were related to the administration of the screening measure itself. For example, although the Spanish version of the PDSS was administered, most Spanish-speaking mothers did not fully complete the screener, or would indicate the same response for all items. Poehlmann et $\mathrm{a}^{22}$ reported that mothers from ethnic and racial minority groups experience depression trajectories significantly different than their White counterparts. It has been suggested that non-White mothers experience higher rates of PPD. ${ }^{34}$ Latina and Black mothers in particular experience more treatment disparities than White mothers. ${ }^{35}$ Future program development and research is needed with ethnic and socioeconomic diversity in $\operatorname{mind}^{22}$ as an additional patientcentered barrier. Thus, given the high frequency of Spanishspeaking mothers not completing the screener in this study, there is further evidence that culturally sensitive approaches are lacking and necessary. For future endeavors, it is imperative to have a bilingual screening team member to improve engagement with this population specifically. Additionally, our experience raises questions about the appropriateness of using the PDSS for this specific population. Previous studies have been successful in Spanish-speaking mothers completing the Spanish versions of the Patient Health Questionnaire-9 and EPDS ${ }^{36}$ in settings outside the NICU, however, very little is known about the Spanish versions of these measures or the PDSS in the NICU setting. Screening and intervention should take place early, particularly for racial and ethnic minority mothers as well as those at high risk due to socioeconomic status. ${ }^{22}$ It is difficult to determine if reasons for low completion rates among Spanish-speaking mothers was due to problems with cultural sensitivity of 
the measure itself and/or specific cultural considerations of Spanish-speaking mothers in the NICU setting. Thus, further study is needed to determine what tools are most appropriate for diverse populations.

Among others, pediatricians have cited time constraints and unfamiliarity of screening instruments as major providercentered barriers to identifying and treating PPD. ${ }^{7}$ It has been suggested that the availability of other providers and length of NICU stay can help offset this time limitation. ${ }^{7}$ However, this was not found to be the case with the current study. Time constraints presented as constant barriers for the nursing staff and study coordinator. Although nurses spend a substantial amount of time with mothers ${ }^{37}$ and have been seen as good sources of support to mothers, ${ }^{20}$ they have many competing demands in caring for the infant patients.

Additional system-centered variables related to the administration of the screening measure were identified as well. Some NICUs have found it helpful to recruit unit-based psychologists; ${ }^{23}$ however, without appropriate financial support, use of mental health and/or social service providers within the hospital system can encounter time limitations and lack of reimbursement. For the current project, mental health providers in particular found it difficult to justify time away from other clinical duties with pressures for sustainability and reimbursement. Reimbursement for services rendered was not feasible due to lack of appropriate billing codes. Although the cost of screening in general is low, programs must consider provider time and absence of means for reimbursement as barriers for sustainability of such programs.

Although there was buy-in from medical and nursing staff, it was difficult obtaining hospital administration support, making it difficult to link the PDSS with routine screening procedures. It has been previously suggested that hospital administration can provide valuable infrastructure from the Quality Assurance/Quality Improvement department for data collection; ${ }^{7}$ this was found to be a significant challenge for the current study.

Given these administrative barriers, several recommendations should be considered when developing a screening program such as the current study. Firstly, from the perspective of patient-centered barriers, the need for culturally sensitive approaches must be recognized. Secondly, when considering provider-centered barriers, programs should consider how they will support and protect time needed from providers to successfully implement such a program. Finally, due to lack of reimbursement feasibility, it is imperative for programs to consider how to justify the cost and sustainability of their programs.

\section{Referral barriers}

Little infrastructure is required for screening and the financial cost of screening itself is minimal; however, the follow-up needs for those mothers requiring referrals can be challenging and present patient-centered barriers. ${ }^{7}$ This study found that referring mothers to community mental health centers did not work for two reasons. First, these mothers did not fit the majority of the population served at these centers. Second, because many of these women lose insurance coverage after 60 days postpartum, the majority of the women did not qualify for services at community mental health centers. Within the current sample, most NICU families had Medicaid as their primary insurance. In the state of Oklahoma, Medicaid coverage for mothers includes pregnancy and extends to 60 days postpartum. When needs were identified, this coverage was expired or close to expiring. Therefore, it was difficult to make appropriate referrals to providers who would accept Medicaid, various private insurances, or no insurance. Many low-income women lack accessibility to general health insurance and eligibility of mental health benefits is even more limited. ${ }^{38}$ Cost and access to mental health providers are significant barriers for some women to seek services from recommended referrals. ${ }^{7}$ Follow-up mental health services from early intervention hospital settings are rare, primarily due to cost. ${ }^{39}$ Although many early intervention programs have been found to be useful, they are primarily time-limited research or foundation funded. ${ }^{38}$ Further policy is needed to address Oklahoma's limited capacity and lack of federal incentives in order to bring research informed practices to scale. ${ }^{38}$

The current study encountered and made attempts to overcome provider-centered and system-centered barriers regarding limited referral options. A common difficulty encountered with positive PPD screen is lack of appropriate referral resources. ${ }^{23}$ Consideration of many providers including obstetrics, family medicine, and behavioral health providers within the hospital, health care system, or community has been previously reported as means of acquiring needed services. ${ }^{7}$ However, within the context of current health care, many challenges are present regarding the utilization of various providers. For example, an imbalance of services available as well as lack of coordination of services can lead to duplication of services or fragmentation of services. ${ }^{40}$ Further, the insurance coverage of many individuals reinforces the utilization of episodic care rather than continuity of care, resulting in limited coordination and communication regarding the needs of the individual. In addition, utilization of episodic care over continuous care can obscure responsibility of providing 
recommendations and follow-up regarding the outcomes of such recommendations. Among those providers who screen for PPD, further documentation of assessment and treatment is frequently absent. ${ }^{19}$ This may be partly due to lack of accountability to the responsibility of making and following-up on recommendations. If no single body or agency can be held responsible for such duties, communication and coordination among providers is imperative.

In response to the concerns raised regarding the coordination of care and follow-up of outcomes, the current study developed the Perinatal Mood and Anxiety Screening for Mothers of NICU Infants Release of Information form. This form facilitated communication of information regarding mothers' screening results via fax to other referral sources, as well as the mothers' primary care physician as a way of closing the information loop. In order to appropriately provide follow-up with mothers, it is vitally important to inform the mothers' primary care physician as well as provide adequate information regarding the care needed.

Additionally, due to difficulties accessing appropriate outpatient mental health services we collaborated with a local Federally Qualified Health Center (FQHC). FQHCs have several qualities that make them an ideal resource. A large number of FQHCs have onsite behavioral health services, many including therapy services and psychotropic medication management. Further, FQHCs provide services to patients with and without insurance and there are several FQHCs throughout Oklahoma. Ensuring appropriate resources for follow-up is imperative when considering developing a screening program. ${ }^{7}$ Having a well-established and feasible plan for referrals can also help decrease liability concerns. ${ }^{7}$

For those considering implementing a universal screening program for PPD in the NICU, barriers regarding access to referrals must be anticipated. Some recommendations for planning to encounter and overcome such barriers include: 1) becoming familiar with resources in the community that may be available to mothers with Medicaid, private insurance, and no insurance; 2) considering multiple types of provider disciplines and settings; 3) having a well-established, yet feasible plan for referrals. One suggestion for addressing these recommendations is to acquire contact with local FQHCs as potential resources. FQHCs offer many opportunities to address the needs of patients who may not otherwise have access to services due to cost and/or availability of services.

\section{Limitations}

Although the current study provided insight into the potential barriers of implementing a universal screening program in the NICU setting, as well as recommendations for those institutions planning to develop such programs, there were several limitations to the study. For example, significant challenges presented in making initial contact with mothers in the NICU. This resulted in protocol changes in efforts to overcome the barriers of not being able to establish contact. The revisions to the protocol make it difficult to tease out whether one method was more feasible or effective than the others. Future studies are needed to evaluate and compare the various types of efforts to make contact (eg, study coordinator alone, nurse alone, educational materials with coordinator follow-up). The results of the current study are limited in gaining insight about what type of approach may be the most efficacious. In addition, not exactly knowing why some women chose not to complete the screener further limits the ability of the current study to make recommendations for improved recruitment.

Further, there were limitations in the current study due to a large number of Spanish-speaking mothers not completing the screening questionnaire. The majority of the mothers who completed the screening measure were Caucasian. Therefore, little is known as to why some ethnic groups were less likely to participate. Additionally, little insight is gained regarding what cultural sensitivities are needed to address the needs of this population. This is of specific concern due to the higher rates of PPD for non-White mothers. ${ }^{34}$ Additionally, it has been suggested that mothers from ethnic and racial minority groups experience depression trajectories significantly different than their White counterparts. ${ }^{22}$ Future research is needed to investigate appropriate means to approach the needs of mothers in the NICU in a culturally sensitive manner.

Also the sample was limited to a single medical center NICU and cannot be generalized to more ethnically or socioeconomically diverse populations or settings. The limitation of generalizability may particularly be of importance to the findings and recommendations regarding referral barriers. Further knowledge is needed regarding the specific referral needs of different ethnic and socioeconomically diverse mothers, as well as the access to such services. Replication at other sites as well as among ethnically diverse populations is needed.

\section{Relevance}

The primary relevance of this study is intended for institutions and providers considering or in the beginning phases of developing and implementing a new program aimed to screen mothers in the NICU for PPD. Mothers of infants in the NICU report significant symptoms of PPD., ${ }^{4,5}$ This study helps to 
confirm the need for routine screening for PPD in the NICU setting and the subsequent, appropriate referrals of these families to mental health professionals. Identifying and addressing maternal stress and depression with developed interventions can help promote family stability, maternal adjustment, and long-term benefits to the infant's development. ${ }^{20}$ There is a great need for PPD screening in the NICU and this has been consistently supported in the literature. ${ }^{7,10,23}$ However, many institutions and providers decide against implementation or try and struggle, due to many barriers encountered.

This article should encourage those who have steered away from implementation and enhance the efforts of the struggling implementers. Development of a PPD screening process in the NICU involves support, training, implementation, and coordination from administrators, medical staff, new mothers, and mental health specialists. Several predictable challenges to program development require ongoing assessment and response to these challenges. The recommendations provided in this article can help raise awareness of barriers that should be anticipated as well as provide some direction in overcoming these barriers.

\section{Acknowledgment}

This project was partially supported by a Mental Health Transformation Grant from the Oklahoma Department of Mental Health and Substance Abuse Services awarded from the Substance Abuse and Mental Health Service Administration.

\section{Disclosure}

The authors report no conflicts of interest in this work.

\section{References}

1. Division of Reproductive Health, National Center for Chronic Disease Prevention and Health Promotion. Atlanta, GA: Centers for Disease Control and Prevention; 2013.

2. Centers for Disease Control and Prevention (CDC). Prevalence of self reported postpartum depressive symptoms-17 states, 2004-2005. MMWR Mob Mortal Wkly Rep. 2008;15(14):361-366.

3. Miller LJ, Rukstalis M. Postpartum Mood Disorders. 1st ed. Washington, DC: American Psychiatry Press; 1999:3-20.

4. Tahirkheli NN, Cherry AS, Tackett AP, McCaffree MA, Gillaspy SR. Postpartum depression on the Neonatal Intensive Care Unit: current perspectives. Int J Womens Health. 2014;6:975-987.

5. Vigod SN, Villegas L, Dennis CL, Ross LE. Prevalence and risk factors for postpartum depression among women with preterm and low-birth-weight infants: a systematic review. BJOG. 2010;117(5):540-550.

6. Alici-Evcimen Y, Sudak DM. Postpartum depression. Prim Care Update Ob Gyns. 2003;10(5):210-216.

7. Mounts KO. Screening for maternal depression in the neonatal ICU. Clin Perinatal. 2009;36:137-152.

8. Carter JD, Mulder RT, Frampton CMA, Darlow BA. Infants admitted to a Neonatal Intensive Care Unit: parental psychological status at 9 months. Acta Paediatr. 2007;96:1286-1289.
9. Amankwaa LC, Pickler RH, Boonmee J. Maternal responsiveness in mothers of preterm infants. Newborn Infant Nurs Rev. 2007; $7(1): 25-30$.

10. Blucker RT, Gillaspy JA, Jackson D, et al. Postpartum depression in the NICU: an examination of the factor structure of the Postpartum Depression Screening Scale. Adv Neonatal Care. 2014; 14(6):424-432.

11. Melnyk BM, Feinstein NF, Alpert-Gillis L, et al. Reducing premature infants' length of stay and improving parents' mental health outcomes with the Creating Opportunities for Parent Empowerment (COPE) Neonatal Intensive Care Unit program: a randomized, controlled trial. Pediatrics. 2006;118:1414-1427.

12. Cusson RM. Factors influencing language development in preterm infants. J Obstet Gynecol Neonatal Nurs. 2003;32:402-409.

13. Melnyk BM, Alpert-Gillis L, Feinstein NF, et al. Improving cognitive development of low-birth-weight premature infants with the COPE program: a pilot study of the benefit of early NICU intervention with mothers. Res Nurs Health. 2001;24:373-389.

14. Pinelli J. Effects of family coping and resources on family adjustment and parental stress in the acute phase of the NICU experience. Neonatal Netw. 2000;19:27-37.

15. Rogers CE, Kidokoro H, Wallendorf M, Inder TE. Identifying mother of very preterm infants at-risk for postpartum depression and anxiety before discharge. J Perinatol. 2013;33:171-176.

16. Shields-Poe D, Pinelli J. Variables associated with parental stress in Neonatal Intensive Care Units. Neonatal Netw. 1997;16(1):29-37.

17. Beck CT. Recognizing and screening for postpartum depression in mothers of NICU infants. Adv Neonatal Care. 2003;3(1):37-46.

18. De Magistris A, Coni E, Puddu M, Zonza M, Fanos V. Screening of postpartum depression: comparison between mothers in the Neonatal Intensive Care Unit and in the neonatal section. J Matern Fetal Neonatal Med. 2010;23(Suppl 3):101-103.

19. Gjerdingen DK, Yawn BP. Postpartum depression screening: importance, methods, barriers, and recommendations for practice. J Am Board Fam Med. 2007;20:280-288.

20. Davis L, Edwards H, Mohay H, Wollin J. The impact of very premature birth on the psychological health of mothers. Early Hum Dev. 2003;73:61-70.

21. Yurdakul Z, Akman I, Kuscu MK, et al. Maternal psychological problems associated with neonatal intensive care admission. Int $J$ Pediatr. 2009;2009:1-7.

22. Poehlmann J, Schwichtenberg AJM, Bolt D, Dilworth-Bart J. Predictors of depressive symptom trajectories in mothers of preterm or low birth weight infants. J Fam Psychol. 2009;23(5):600-704.

23. Hynan MT, Mounts KO, Vanderbilt DL. Screening parents of highrisk infants for emotional distress: rationale and recommendations. J Perinatol. 2013;33:748-753.

24. Earls MF, Committee on psychological aspects of child and family health American Academy of Pediatrics. Incorporating recognition and management of perinatal and postpartum depression into pediatric practice. Pediatrics. 2010;126(5):1032-1039.

25. Hearn G, Iliff A, Jones I, et al. Postnatal depression in the community. Br J Gen Pract. 1998;48:1064-1066.

26. Georgiopoulos AM, Bryan TL, Wollan P, Yawn BP. Routine screening for postpartum depression. J Fam Pract. 2001;50:117-122.

27. Evins GG, Theofrasstous JP, Galvin SL. Postpartum depression: a comparison of screening and routine clinical evaluation. Am J Obstet Gynecol. 2000;182:1080-1082.

28. Katon WJ, Ludman EJ. Improving services for women with depression in primary care settings. Psychol Women Quarterly. 2003;27:114-120.

29. Scholle SH, Haskett RF, Janusa BH, Pincus HA, Kupfer DJ. Addressing depression in obstetrics/gynecology practice. Gen Hosp Psychiatry. 2003;25:83-90.

30. Olson AL, Kemper KJ, Kellerher KJ, Hammond CS, Zuckerman BS, Dietrich AJ. Primary care pediatricians' roles and perceived responsibilities in the identification and management of maternal depression. Pediatrics. 2002;110:1169-1176. 
31. Beck CT, Gable RK. Postpartum Depression Screening Scale Manual. Los Angeles, CA: Western Psychological Services; 2002.

32. Zubaran C, Schumacher M, Roxo M, Foresti K. Screening tools for postpartum depression: validity and cultural dimensions. Afr J Psychiatry. 2010;13:357-365.

33. McCabe K, Blucker R, Gillaspy JA, et al. Reliability of the Postpartum Depression Screening Scale in the Neonatal Intensive Care Unit. Nurs Res. 2012;61(6):441-445.

34. Howell EA, Mora PA, Horowitz CR, Leventhal H. Racial and ethnic differences in factors associated with early postpartum depressive symptoms. Obstet Gynecol. 2005;105:1442-1450.

35. Zittel-Palamara K, Rockmaker JR, Schwabel KM, Weinstein WL, Thompson SJ. Desired assistance versus care received for postpartum depression: access to care differences by race. Arch Womens Ment Health. 2008;11:81-92.
36. Yawn BP, Dietrich AJ, Wollan P, et al. TRIPPD: a practice-based network effectiveness study of postpartum depression screening and management. Ann Fam Med. 2012;10:320-329.

37. Silverstein M, Feinberg E, Hegel M. Preventing depression of mothers of preterm infants. Psychiatr Serv. 2011;62(1):101.

38. Knitzer J, Theberge S, Johnson K. Reducing Maternal Depression and its Impact on Young Children Toward a Responsive Early Childhood Policy Framework. New York, NY: Columbia University; 2008.

39. Barry LM, Singer GHS. Reducing maternal psychological distress after the NICU experience through journal writing. J Early Interv. 2001;24(4):287-297.

40. Van Mullen C, Conway AE, Mounts K, Weber D, Browning CA. Regionalization of perinatal care in Wisconsin: a changing health care environment. WMJ. 2004;103(5):35-38.
Journal of Multidisciplinary Healthcare

\section{Publish your work in this journal}

The Journal of Multidisciplinary Healthcare is an international, peerreviewed open-access journal that aims to represent and publish research in healthcare areas delivered by practitioners of different disciplines. This includes studies and reviews conducted by multidisciplinary teams as well as research which evaluates the results or conduct of such teams or

\section{Dovepress}

healthcare processes in general. The journal covers a wide range of areas and welcomes submissions from practitioners at all levels, from all over the world. The manuscript management system is completely online and includes a very quick and fair peer-review system. Visit http://www.dovepress.com/testimonials.php to read real quotes from published authors.

Submit your manuscript here: http://www.dovepress.com/journal-of-multidisciplinary-healthcare-journal 